\title{
Schutz oder Eigenverantwortung?
}

\section{Verbraucherpolitik muss in Zielsetzung und Handeln vor dem Hintergrund gesellschaftlichen Wandels bestehenden und neven Verbraucherbedïrfnissen gerecht werden. Schutz und Eigenverantwortlichkeit der Verbraucher sind die zentralen Ausgangspunkte aller Maßnahmen. Langfristiges Ziel: Mehr Nach- haltigkeit im Konsumverhalten.}

\section{$\mathrm{V}$} Von Matthias Berninger
erbraucherpolitik wirkt in bereiche der Verbraucherinnen und Verbraucher hinein und muss auf gesellschaftliche Veränderungen reagieren. Viele Bedürfnisse werden heutzutage durch den Kauf von Gütern und die Inanspruchnahme von Dienstleistungen gedeckt. Im Zeitablauf unterliegt dabei die Nachfrage der Verbraucherinnen und Verbraucher erheblichen Veränderungen. So entfällt auf Reisen, Kommunikation und Freizeit mittlerweile ein beachtlicher Teil der privaten Ausgaben und in den Bereichen Altersvorsorge, Pflege und Gesundheit entstehen neue Bedürfnisse und neue Märkte. Angesichts der neuen und vielfältigen Möglichkeiten fällt es Verbraucherinnen und Verbrauchern oftmals schwer, die Leistungen der Anbieter zu vergleichen und bedarfsgerechte Angebote zu finden. Verbraucherinnen und Verbraucher wollen als Marktbeteiligte auf ein angemessenes Schutzniveau vertrauen können und Zugang zu anbieterunabhängiger Information erhalten, um sich mit neuen Produkten und in neuen Märkten zurecht zu finden. Der Verbraucherpolitik stellen sich in dieser Situation zwei Aufgaben:

- den vorsorgenden Schutz von Gesundheit, Sicherheit und wirtschaftlichen Interessen sowie den Schutz vor Täuschung und Irreführung der Verbraucherinnen und Verbraucher zu gewährleisten und

- selbstbestimmte Entscheidungen zu ermöglichen.

Verbraucherpolitik hat seit der Vollendung des Europäischen Binnenmarktes eine europäische, mit fortschreitender Globalisierung eine weltweite Dimension. Die Politik ist gefordert, auf der nationalen und internationalen Ebene Rahmenbedingungen für eine Entwicklung zu setzen, die Verbraucherinteressen angemessen berücksichtigt. So ist den Erfordernissen des Verbraucherschutzes in der Europäischen Union bei der Fest- legung und Durchführung der Gemeinschaftspolitiken Rechnung zu tragen. Dies wurde ausdrücklich 1998 im Vertrag von Amsterdam festgelegt.

Spannungsfelder der Verbraucherpolitik ergeben sich aus der Frage, wie und mit welchen Zielen Probleme gelöst werden, ob staatliche Eingriffe erforderlich oder andere Lösungswege möglich sind. Damit sind Eigenverantwortung der Verbraucherinnen und Verbraucher und staatliche Regulierung als zwei Pole zu definieren. Verzichtet der Staat auf Regulierung und zieht sich zurück, erhöhen sich automatisch die Anforderungen an die Verbraucher, die sich mit neuen Situationen auseinander setzen müssen. Greift der Staat zu stark ein, besteht die Gefahr der Bevormundung und der Überregulierung. Die Verbraucherpolitik hat in der modernen Dienstleistungsund Industriegesellschaft eine vor allem vorsorgende Funktion und trägt zum Funktionieren der Märkte bei (1).

\section{Schutz von Gesundheit und Sicherheit}

Der vorsorgende Schutz von Gesundheit und Sicherheit ist eine klassische staatliche Aufgabe. Der Staat muss im Bereich der Lebensmittel- und Produktsicherheit den rechtlichen Rahmen set-

Einen Überblick über alle bisher erschienenen Hefte des Informationsdienstes "Ökologisches Wirtschaften" finden Sie auf unseren Internetseiten unter der Adresse:

\section{http://www.oekom.de}

Wir freuen uns auf Ihren Besuch! zen. Die Maxime ist, dass Lebensmittel und Produkte sicher und gesundheitlich unbedenklich sein müssen. National wie auch auf internationaler Ebene setzt sich die Bundesregierung dafür ein, dass ein hohes Schutzniveau für die Verbraucher gewährleistet wird. Dabei macht sich die Bundesregierung das Vorsorgeprinzip zu eigen, das heißt bei Unsicherheiten in der wissenschaftlichen Bewertung und Unklarheit über das Ausmaß gesundheitlicher Gefährdungen werden nach Maßgabe der Verhältnismäßigkeit Maßnahmen ergriffen. Durch das frühzeitige Erkennen potenzieller Gefahren können unkalkulierbare Risiken vermieden werden.

Neben gesetzlicher Regulierung, privatwirtschaftlich organisierter Normierung und Standardisierung zur Erhöhung der Produktsicherheit sowie präventiv wirkender Haftungsregelungen setzt das Bundesministerium für Verbraucherschutz, Ernährung und Landwirtschaft (BMVEL) auch auf Dialog und Kooperation. Am Beispiel von Acrylamid als einem möglicherweise gesundheitsgefährdenden Stoff in Lebensmitteln zeigt sich die Vorteilhaftigkeit von Kooperation. Durch das gemeinsam mit der Lebensmittelwirtschaft erarbeitete Minimierungskonzept konnte der Acrylamidgehalt in verarbeiteten Lebensmitteln deutlich gesenkt werden. Ähnliche Erfahrungen hat das BMVEL bereits anlässlich des BSE-Skandals im Rahmen des „Magischen Sechsecks“, also dem engen Zusammenwirken von Landwirtschaft, Vorleistungsbereich, Ernährungswirtschaft, Lebensmitteleinzelhandel, Verbrauchern und Politik, gemacht. Wir werden darauf achten, dass regelmäßig neueste wissenschaftliche Erkenntnisse in die Risikobewertung und das Risikomanagement einfließen.

Das Beispiel von Acrylamid zeigt aber auch, wie wichtig die Eigenverantwortung der Verbraucherinnen und Verbraucher sein kann. Durch die Art und Weise, wie sie Lebensmittel im Haushalt zubereiten, bestimmen sie selbst über den Grad der Risikominimierung. Damit Verbraucherinnen und Verbraucher sich sachgerecht informieren können, unterstïtzt das BMVEL vielfältige Informationsmaßnahmen.

\section{Schutz der wirtschaftlichen Interessen}

Staatliche Aufgabe im Bereich des Schutzes wirtschaftlicher Verbraucherinteressen ist es, für einen fairen Interessenausgleich zwischen allen Marktbeteiligten zu sorgen. Angesichts dynamischer Marktentwicklungen und durch Liberalisierung entstehender Märkte ist dies eine anspruchsvolle 
Daueraufgabe. Die Verbraucherinnen und Verbraucher müssen in die Lage versetzt werden, im Verhältnis zur Anbieterseite ihre berechtigten Interessen effektiv wahrnehmen zu können.

Dazu ist es erforderlich, die bestehenden rechtlichen Regelungen zum Schutz der wirtschaftlichen Interessen zu überprüfen und zu verbessern. So wird beispielsweise mit der Reform des Gesetzes gegen den unlauteren Wettbewerb die Rolle des Verbrauchers als Marktteilnehmer gestärkt. Der Schutz der Verbraucher wird gleichberechtigt neben den Schutz der Mitbewerber und der sonstigen Marktteilnehmer gestellt.

Bei der Bekämpfung des Missbrauchs von 0190er/0900er-Mehrwertdiensterufnummern gilt es ebenfalls, die Rolle des Verbrauchers als Marktteilnehmer zu stärken und ihn vor betrügerischen Handlungen zu schützen. Grundsätzlich sind technologische Entwicklungen zur einfachen Bezahlung von Mehrwertdiensten zu begrüßen; von Seiten des Staates muss aber darauf geachtet werden, dass Verbraucherinnen und Verbraucher vor Nutzung der Angebote sich ein Bild über die entstehenden Kosten sowie ihre Rechte machen können. So sind in dem Gesetzentwurf zur Bekämpfung des Missbrauchs solcher Bezahlsysteme unter anderem die Einführung von Entgeltobergrenzen und eine Preisangabenpflicht vor Inanspruchnahme der Dienste vorgesehen.

Auch in neuen Märkten wie dem Internet-Handel oder neuen Produkten bei Finanzdienstleistungen ist ein hohes Niveau an Verbraucherschutz unabdingbar. Dies betrifft zum einen die Möglichkeit, bei bestimmten Mängeln oder Pflichtverletzungen von Verträgen zurückzutreten und zum anderen vor Irreführung und Täuschung geschützt zu sein. Nur wenn die Verbraucher sich auf gewisse Standards, sei es durch Rechtsgrundlagen oder durch effektive Selbstregulierung, verlassen können, entsteht Vertrauen als unerlässliche Basis für die Funktionsfähigkeit und die Entwicklung von Märkten.

Verbrauchervertrauen entsteht auch durch verbesserte Informationen und erhöhte Markttransparenz. Insofern sind die von verschiedenen Seiten vorgebrachten Einwände gegen verbesserte Informationsrechte nicht verständlich. Auch das Bundesverfassungsgericht hat in seinem Beschluss über die Berechtigung der Bundesregierung zur Informationsarbeit im Bereich des Verbraucherschutzes bestätigt, dass ein hohes Maß an Markttransparenz zur Funktionsfähigkeit von Märkten beiträgt. Zur Verbesserung der Transparenz und um dem Recht der
Verbraucherinnen und Verbraucher auf Information zu entsprechen, werden wir zu einem neuen Anlauf für das Verbraucherinformationsgesetz ansetzen.

\section{Eigenverantwortung und Nachhaltigkeit}

Zukunftsvorsorge heißt auch, dass Konsumenten sich über die Folgen ihrer Entscheidungen bewusst werden. Jede Marktbeteiligung der Verbraucher beeinflusst auch die Investitionsentscheidungen der Anbieter. Wenn in einer freiheitlichen Gesellschaft die Anbieter das Vorschlagsrecht für ihre Angebote haben, so haben die Verbraucherinnen und Verbraucher das Recht, auf dieses Angebot einzugehen oder auch nicht. Insofern bilden Märkte einen Entdeckungsprozess ab, dessen Ergebnis auf den Entscheidungen der Marktakteure beruht, das aber vor Ablauf des Prozesses letztlich unbekannt ist.

Aus verschiedenen Studien der Konsumentenforschung wissen wir, dass Verbraucher zunehmend bereit sind, in ihre Konsumentscheidungen ethische und ökologische Überlegungen einfließen zu lassen. Die Entscheidungskriterien gehen somit weit über die eigentliche Produktqualität hinaus hin zur Beurteilung von Produktions- und Handelsprozessen. Verbraucherinnen und Verbraucher möchten auch darüber informiert werden, wo und unter welchen Bedingungen die Waren und Dienstleistungen erstellt werden. In den Bereichen, in denen es abzusehen ist, dass der Markt auf entsprechende Bedürfnisse der Verbraucher nicht angemessen reagiert, kann Verbraucherpolitik dazu beitragen, Hemmnisse, Barrieren und soziale Fehlentwicklungen abzubauen.

Hier sehen wir eine Aufgabe des Staates, gesellschaftlich erwünschte Entwicklungen zu unterstïtzen. In Johannesburg hat sich die Weltgemeinschaft verpflichtet, das Produktions- und Konsumverhalten zu verändern, um eine nachhaltige Entwicklung zu erreichen. Die Industriestaaten wollen hierbei eine führende Rolle übernehmen. Ein 10-Jahres-Rahmenprogramm soll unter Berücksichtigung der Leistungsfähigkeit der Ökosysteme durch verbesserte Effizienz eine Entkopplung von wirtschaftlichem Wachstum und Umweltverbrauch ermöglichen. Daneben soll durch Aufklärung und Information ein verantwortungsvoller Konsum gefördert werden. Die Bundesregierung wird Maßnahmen unterstïtzen, die den Verbrauchern helfen, ihr Verhalten an den Kriterien der Nachhaltigkeit auszurichten. So soll der vom Nachhaltigkeitsrat der Bundesregierung exemplarisch entwickelte „Nachhaltige Warenkorb“ als Dialoginstrument genutzt und weiterentwickelt werden.

Positive Impulse für eine Ausrichtung des Konsums an Nachhaltigkeitskriterien setzen wir auch mit der Initiierung und Unterstützung entsprechender Qualitätszeichen. Die Erfahrungen mit dem Bio-Siegel zeigen, dass mit eindeutigen, für den Verbraucher leicht erkennbaren und vertrauenswürdigen Zeichen eine wesentliche Verbesserung der Markttransparenz gelingt. Bei einer transparenten und verlässlichen Orientierungshilfe finden sich Verbraucher auf den Märkten besser zurecht. Auch Zeichen für Fair Trade, nachhaltige Forstwirtschaft oder Fischerei können Markttransparenz fördern und eigenverantwortlichen Konsum ermöglichen.

In einer sozialen Marktwirtschaft legt der Staat die Spielregeln fest und sorgt für deren Anpassung und Einhaltung. Wie beim Sport ist die Gewährleistung von Schutzniveaus und das Funktionieren von Märkten nur dann gegeben, wenn die Spielregeln eingehalten werden. Wenn unfaires Verhalten bestraft wird, kann im Wettbewerb wie auch im Sport der Bessere gewinnen. Dies ist im Interesse der Verbraucher, aber auch der Unternehmen. Aufgabe der Politik im 21. Jahrhundert wird sein, Wohlstand und Lebensqualität unter der Prämisse der nachhaltigen Entwicklung zu fördern.

\section{Anmerkungen:}

(1) Im Aktionsplan Verbraucherschutz, der am 7. Mai 2003 vom Bundeskabinett beschlossen wurde, werden die verbraucherrelevanten Vorhaben und Ziele der Bundesregierung aufgezeigt. Er umfasst über alle Politikbereiche hinweg konkret geplante Rechtsetzungsvorhaben oder Aktionen für diese Legislaturperiode.

Download: www.verbraucherministerium.de

\section{Der Autor}

Matthias Berninger ist Parlamentarischer Staatssekretär im Bundesministerium für Verbraucherschutz, Ernährung und Landwirtschaft (BMVEL).

Kontakt: BMVEL, 11055 Berlin.

Tel. 01888-529-3186 
(c) 20I0 Authors; licensee IÖW and oekom verlag. This is an article distributed under the terms of the Creative Commons Attribution Non-Commercial No Derivates License (http://creativecommons.org/licenses/by-nc-nd/3.o/), which permits unrestricted use, distribution, and reproduction in any medium, provided the original work is properly cited. 